\title{
Association of beta 2 adrenoceptor gene polymorphisms in Malaysian hypertensive subjects
}

\author{
M. Komara ${ }^{1}$, R. Vasudevan ${ }^{2}$, P. Ismail ${ }^{1}$, S.A. Bakar ${ }^{1}$, S.R. Pishva ${ }^{1}$ and \\ F. Heidari ${ }^{1}$ \\ ${ }^{1}$ Genetic Research Group, Department of Biomedical Science, \\ Faculty of Medicine and Health Sciences, Universiti Putra Malaysia, \\ Selangor, Malaysia \\ ${ }^{2}$ Institute of Gerontology, Universiti Putra Malaysia, Serdang, \\ Selangor, Malaysia \\ Corresponding author: P. Ismail \\ E-mail: patimahismail@gmail.com
}

Genet. Mol. Res. 13 (2): 2939-2948 (2014)

Received January 3, 2013

Accepted July 31, 2013

Published April 16, 2014

DOI http://dx.doi.org/10.4238/2014.April.16.2

\begin{abstract}
The sympathetic nervous system plays a major role in blood pressure regulation. Beta $2(\beta 2)$ adrenoceptor gene polymorphisms have been associated with hypertension in different populations with conflicting results. We examined the association of three common polymorphisms, Arg16Gly, Gln27Glu, and Thr164Ile, of the $\beta 2$ adrenoceptor gene in Malaysian hypertensive subjects. A total of 160 hypertensive and control subjects were recruited. Systolic blood pressure (SBP), diastolic blood pressure (DBP), and anthropometric measurements were obtained from each subject. Biochemical analyses of lipid profiles were conducted with an autoanalyzer. DNA samples were extracted from blood and buccal cells. Genotyping was accomplished with polymerase chain reaction-restriction fragment length polymorphism. SBP, DBP, body mass index, and biochemical factors all differed significantly between case and control subjects
\end{abstract}


$(\mathrm{P}<0.05)$. The genotype frequencies of Arg16Arg, Arg16Gly, and Gly16Gly were $22.5,70$, and $7.5 \%$ among cases and 33.1, 63.1, and $3.8 \%$ among controls, respectively. The genotype frequencies of Gln27Gln, Gln27Glu, and Glu27Glu among cases were 41.1, 50, and $1.9 \%$ compared to $77.5,20.6$, and $1.9 \%$ among controls, respectively. In this study, the Gln27Glu polymorphism was significantly associated with Malaysian hypertensive subjects $(\mathrm{P}<0.05)$. Therefore, the Gln27Glu polymorphism of the $\beta 2$ adrenoceptor could be a risk factor associated with hypertension among Malaysians.

Key words: Gln27Glu polymorphism; $\beta 2$ adrenoceptor; PCR-RFLP; Hypertension

\section{INTRODUCTION}

Essential hypertension is a complex disease influenced by both genetic and environmental factors. Several candidate genes have been hypothesized to play role in the development of hypertension, including the $\beta 2$ adrenoceptor gene (Agarwal et al., 2005). The sympathetic nervous system plays a major role in blood pressure regulation. The $\beta 2$ adrenoceptor is a G-protein coupled receptor that, upon activation by catecholamine, increases the intracellular second messenger cyclic adenosine monophosphate (cAMP). $\beta 2$ adrenoceptor mediates the vasodilatation that is important in blood pressure control. Other blood pressure regulating effects of the $\beta 2$ adrenoceptor include renal sodium handling and control of renin release (Pereira et al., 2003). The $\beta 2$ adrenoceptor gene (ADRB2) is a 1242-bp intronless gene, which is located on the long arm of chromosome 5 (5q31-q32). Three common single nucleotide polymorphisms (SNPs) of $A D R B 2$ have been characterized that are functionally important and vastly studied in many populations (Drysdale et al., 2000; Kato et al., 2001): arginine substituted by glycine at nucleotide 16 (Arg16Gly/rs1042714), glutamine substitution by glutamic acid at nucleotide 27 (Gln27Glu/rs1042714), and threonine substitution by isoleucine (Thr164Ile/rs1800888) at nucleotide 164. In vitro studies demonstrated that the Gly16 $\beta 2$ adrenoceptor variant showed enhanced agonist-promoted downregulation, whereas the Glu27 variant demonstrated reduced agonist-induced downregulation. The Ile164 variant demonstrated decreased receptor responsiveness to agonist stimulation (Garland and Biaggioni, 2001; Leineweber and Brodde, 2004). Numerous studies have attempted to determine the association of these $\beta 2$ adrenoreceptor variants with hypertension and related conditions, revealing conflicting results in different populations (Kotanko et al., 1997; Large et al., 1997; Candy et al., 2000; Masuo et al., 2005; Sethi et al., 2005). Masuo et al. (2005) demonstrated that individuals with Gly16 and Glu27 alleles showed higher blood pressure and weight gain in a cohort study of the Japanese population. Similar findings were observed in a cross-sectional study of the general population of Brazil (Pereira et al., 2003). The164Ile variant was associated with risk of elevated blood pressure in women of the Danish population (Sethi et al., 2005). In contrast, there was no association between $\beta 2$ adrenergic receptor variants and blood pressure in Japanese (Kato et al., 2001) and black African (Candy et al., 2000) populations. These conflicting results motivated us to determine the association of $\beta 2$ adrenoceptor gene polymorphisms in Malaysian hypertensive subjects. 


\section{MATERIAL AND METHODS}

\section{Subjects}

Prior to commencing this study, ethical clearance was sought from the Ethics Committee of the Faculty of Medicine and Health Science, Universiti Putra Malaysia (UPM). Additional permission was obtained from the Seremban Hospital for sample collection. A total of 175 patients were approached, and 160 hypertensive subjects were recruited; the rest of the samples were excluded based on their extreme values and inconsistent results. Hypertensive subjects were recruited based on a diagnosis of hypertension or being on antihypertensive medication. A total of 160 controls were recruited who had never been diagnosed with hypertension and whose averages of three blood pressure readings were lower than 140/90 $\mathrm{mmHg}$. Demographic factors such as age, gender, and race were recorded. Systolic blood pressure (SBP), diastolic blood pressure (DBP), and body mass index (BMI) were obtained from each participant. To determine lipid profiles, 4-5 mL blood was collected from each participant. The biochemical analysis was performed using a Hitachi Autoanalyzer (Hitachi, Mannheim, Germany). Buccal cells were collected from each subject for genomic DNA extraction using a sterile cytological brush.

\section{Biochemical analysis}

Prior to DNA extraction, serum was separated from the blood by centrifugation at $4000 \mathrm{rpm}$ and stored at $-20^{\circ} \mathrm{C}$ until further analysis. Serum levels of high-density lipoproteincholesterol (HDL-C), total cholesterol (TC), and triglycerides (TG) were measured enzymatically on a Hitachi-912 Autoanalyzer with kits supplied by Roche Diagnostics (Mannheim, Germany). Low density lipoprotein cholesterol (LDL-C) was calculated using the Friedewald formula (Friedewald et al., 1972).

\section{Genotyping}

For hypertensive subjects, DNA was extracted from buccal cells using the Puregen Buccal cell kit (Qiagen, Germany), whereas for controls, DNA was extracted from peripheral blood using the Analytikagena kit (Analytikagena, Germany). The extracted DNA was quantified using a spectrophotometer. Polymerase chain reaction (PCR) was carried out using the respective primers and restriction enzymes listed in Table 1. The amplified PCR products of Arg16Gly, Gln27Glu, and Thr164Ile were digested with the BsrDI, ItaI, and Mn1I restriction enzymes, respectively. The fragments were resolved by electrophoresis on $2.5-3 \%$ agarose gel. The gels were stained using Gel Red (Biotium, USA) before visualization with the UV light alpha imager (Alpha Innotech, USA)

\section{Statistical analysis}

Statistical analysis was carried out using the SPSS software version 16.0 (SPSS, USA) for Microsoft Windows. Continuous data were analyzed using the Student $t$-test and analysis of variance (ANOVA). Allele frequencies were compared using the gene counting method. Genotype frequencies were determined by the chi-squared test with two degrees of freedom. A level of $\mathrm{P}<0.05$ was considered to be statistically significant. 
M. Komara et al.

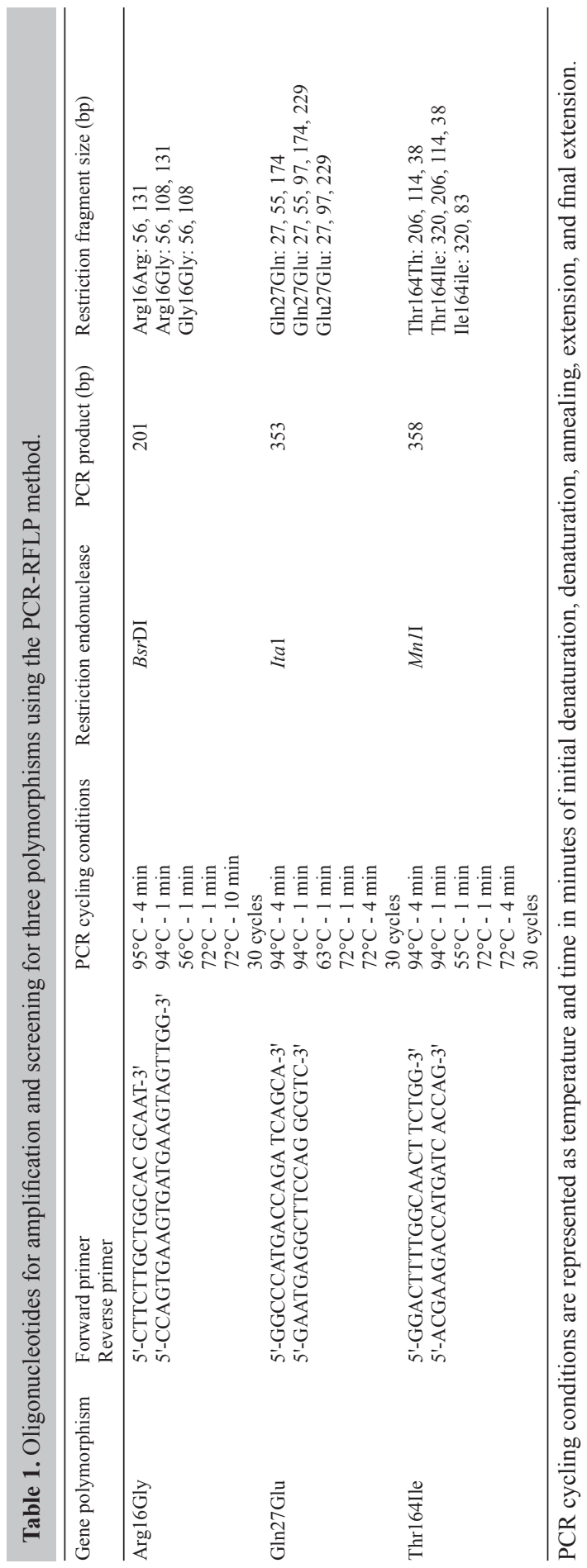




\section{RESULTS}

The initial sample of this study consisted of 338 subjects of whom 18 individuals were excluded due to incomplete information and extreme values. Therefore, a total of 320 subjects were included in this study, consisting of 160 cases and 160 control subjects. Over half of the subjects $(57.2 \%)$ were females and $42 \%$ were males. The majority of the participants in this study were Malays (45.3\%) followed by Chinese $(31.9 \%)$ and Indians $(22.8 \%)$. The average readings of SBP and DBP were $137.12 \pm 21.06$ and $80.35 \pm 9.93 \mathrm{mmHg}$, respectively. Based on anthropometric measurements, the mean BMI of all subjects was calculated as 26.19. Moreover, the biochemical analysis of lipid profiles revealed mean values for TC, LDL, HDL, and TG of $4.89 \pm 1.22,2.97 \pm 1.01,1.17 \pm 0.41$, and $1.38 \pm 0.67 \mathrm{mM}$, respectively. Table 2 shows the baseline characteristics of all subjects. There was a significant difference in the means of age, SBP, DBP, TC, LDL, HDL, and TG between cases and controls ( $\mathrm{P}<0.001)$. Table 3 shows the genotype and allele frequencies of the Arg16Gly and Gln27Glu polymorphisms. Deviations from Hardy-Weinberg equilibrium were tested in both the case and control groups with the chi-squared test. Gln27Glu genotype frequencies were in Hardy-Weinberg equilibrium in both cases and controls $(\mathrm{P}>0.05)$. However, Arg16gly genotypes did not meet the Hardy-Weinberg assumptions in both cases and controls $(\mathrm{P}<0.001)$. All genotypes were determined using PCR-restricted fragment length polymorphism (RFLP) with selected restriction enzymes. In order to genotype the Arg16Gly polymorphism of $A D R B 2$, the amplified PCR product was digested with the $B s r$ DI restriction enzyme. Figure 1 shows the restricted fragments obtained. Genotypes of the Gln27Glu polymorphism were determined by digestion of the PCR product with the ItaI restriction enzyme (Figure 2), while the Thr164Ile polymorphism was determined by incubating the PCR product with the Mn1I restriction enzyme. The fragments were resolved on 3\% agarose gel and showed 38-, 114-, and 206-bp fragments in Thr164Thr homozygotes (Figure 3). All of the 320 samples were homozygous for the wildtype Thr164Thr.

We also examined the relationship between $\beta 2$ adrenoceptor gene polymorphisms and clinical characteristics of the subjects (Table 4). One-way ANOVA showed no significant associations between clinical characteristics and genotypes of the Arg16Gly polymorphism (P $>0.05)$. However, the post hoc analysis of ANOVA showed that the Gln27Glu polymorphism was significantly associated with SBP $(\mathrm{P}<0.05)$.

Table 2. Baseline characteristics of the subjects.
\begin{tabular}{lccc}
\hline Parameters & Cases $(\mathrm{N}=160)$ & Controls $(\mathrm{N}=160)$ & $\mathrm{P}$ \\
\hline Gender (M/F) & $80(50 \%) / 80(50 \%)$ & $57(35.6 \%) / 103(64.4)$ & - \\
Age (years) & $58.31 \pm 11.03$ & $39.21 \pm 13.18$ & $* *$ \\
SBP $(\mathrm{mmHg})$ & $148.36 \pm 20.38$ & $125.81 \pm 14.71$ & $* *$ \\
DBP $(\mathrm{mmHg})$ & $83.22 \pm 9.63$ & $77.30 \pm 9.36$ & $* *$ \\
BMI (kg/m $)$ & $27.21 \pm 4.88$ & $3.17 \pm 5.26$ & $* *$ \\
LDL (mM) & $2.71 \pm 0.94$ & $1.10 \pm 0.35$ & $* *$ \\
HDL (mM) & $1.24 \pm 0.045$ & $1.23 \pm 0.65$ & $* *$ \\
Triglycerides (mM) & $1.51 \pm 0.66$ & $5.25 \pm 1.21$ & $* *$ \\
Total cholesterol (mM) & $4.53 \pm 1.11$ & $14(8.75 \%) / 146(91.25 \%)$ & $*$ \\
Smoker/nonsmoker & $26(16.25 \%) / 134(83.75 \%)$ & $42(26.25 \%) / 118(73.75 \%)$ & $* *$ \\
Family history of hypertension & $77(48.12 \%) / 83(51.87 \%)$ & $*$ \\
(positive/negative) &
\end{tabular}

Continuous data are reported as means \pm standard deviation; categorical data are reported as percentage. $* \mathrm{P}<0.05$; $* * \mathrm{P}<0.01 . \mathrm{NS}=$ not significant. 
Table 3. Genotype and allele frequencies of Arg16Gly and Gln27Glu polymorphisms.

\begin{tabular}{lcr}
\hline Genotype & Cases $(\mathrm{N}=160)$ & Controls $(\mathrm{N}=160)$ \\
\hline Arg16Arg & $36(22.5 \%)$ & $53(33.1 \%)$ \\
Arg16Gly & $112(70 \%)$ & $101(63.1 \%)$ \\
Gly16Gly & $12(7.5 \%)$ & $6(3.8 \%)$ \\
Significance & 0.055 & $207(64.7 \%)$ \\
Alleles & & $113(35.3 \%)$ \\
Arg16 & $184(57 \%)$ & \\
Gly16 & $136(42.5 \%)$ & $124(77.5 \%)$ \\
Significance & 0.062 & $33(20.6 \%)$ \\
Odds ratio $(95 \% \mathrm{CI})$ & $0.739(0.537-1.016)$ & $3(1.9 \%)$ \\
Gln27Gln & $77(48.1 \%)$ & \\
Gln27Glu & $80(50 \%)$ & $281(87.81 \%)$ \\
Glu27Glu & $3(1.9 \%)$ & $39(12.19 \%)$ \\
Significance & 0.000 & \\
Alleles & & \\
Gln27 & $234(73.12 \%)$ & \\
Glu27 & $86(26.88 \%)$ & 0.000 \\
Significance & $0.37(0.249-0.573)$ & \\
Odds ratio $(95 \% \mathrm{CI})$ & &
\end{tabular}

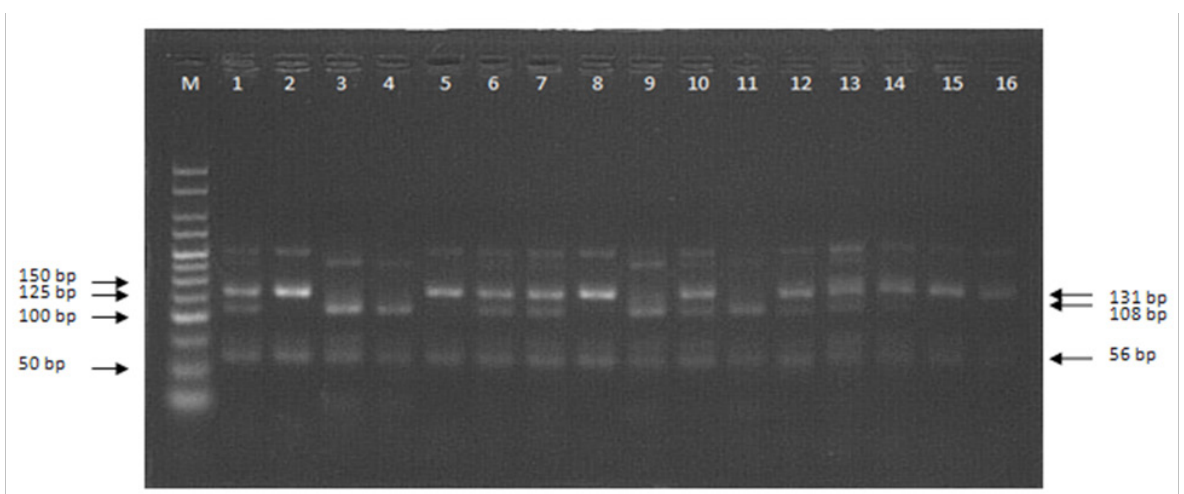

Figure 1. Detection of the Arg16Gly polymorphism by PCR-RFLP. Lane $M=$ hyper-ladder V; lanes 1, 6, 7, 10 , 12,13 , and $14=\operatorname{Arg} 16$ Gly heterozygote. Lanes 2, 5, 8, 15, and 16=Arg16Arg homozygote. Lanes 3, 4, 9 , and 11 $=$ Gly16Gly homozygote.

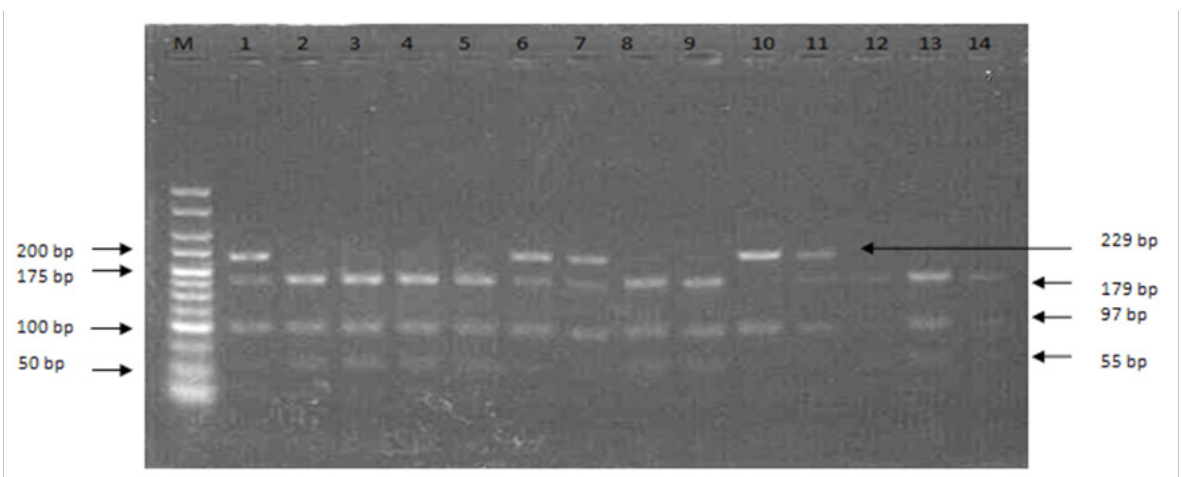

Figure 2. Detection of the Gln27Glu polymorphism by PCR-RFLP. Fragments are resolved on $2.5 \%$ agarose gel. Lane $M=$ hyper-ladder V. Lanes 1, 6, 7, and $11=\mathrm{Gln} 27 \mathrm{Glu}$ heterozygote. Lanes 2, 3, 4, 5, 8, 9, 12, 13, and $14=$ Gln27 homozygote. Lane $10=$ Glu27 homozygote. 


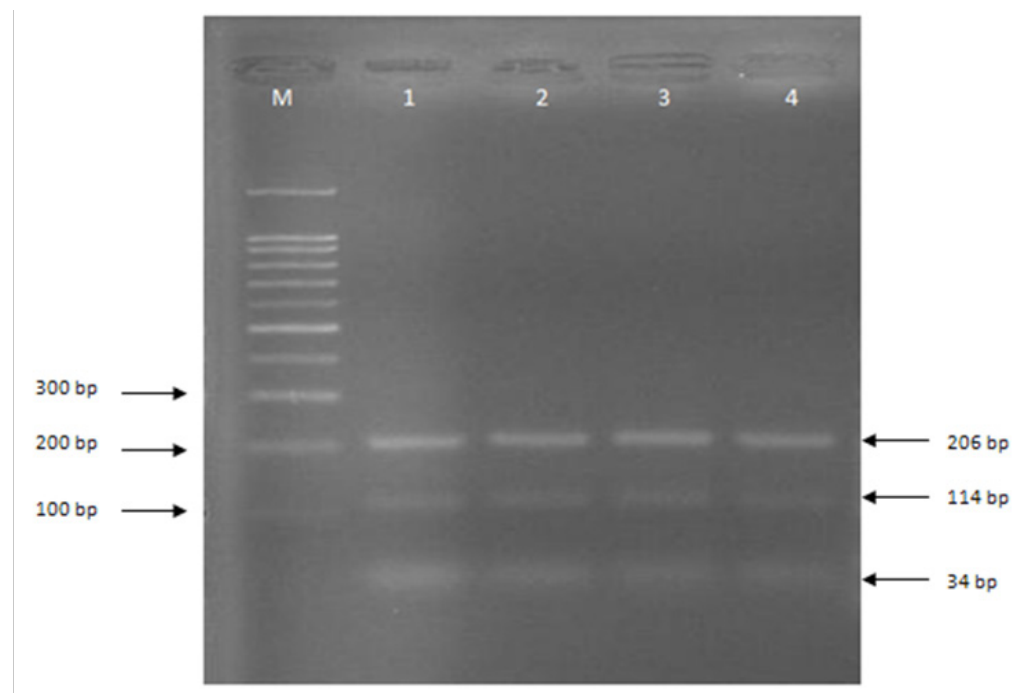

Figure 3. Detection of the Thr164Ile polymorphism by PCR-RFLP. Fragments are resolved on 3\% agarose gel. Lane $M=100$-bp DNA marker. Lanes 1, 2, 3, and $4=$ Thr164Thr homozygote.

Table 4. Clinical characteristics of Arg16Gly and Gln27Glu polymorphisms.

\begin{tabular}{|c|c|c|c|c|c|c|c|c|}
\hline Parameter & Arg16Arg & Arg16Gly & Gly16Gly & $\mathrm{P}$ & Gln27Gln & Gln27Glu & Glu27Glu & $\mathrm{P}$ \\
\hline BMI $\left(\mathrm{kg} / \mathrm{m}^{2}\right)$ & $25.69 \pm 4.57$ & $26.41 \pm 5.50$ & $26.02 \pm 4.62$ & NS & $26.12 \pm 5.28$ & $26.37 \pm 5.22$ & $25.39 \pm 2.37$ & NS \\
\hline $\mathrm{SBP}(\mathrm{mmHg})$ & $133.79 \pm 20.88$ & $138.55 \pm 20.97$ & $137.11 \pm 21.06$ & NS & $134.39 \pm 20.9$ & $141.65 \pm 20.56$ & $142.83 \pm 23.89$ & $*$ \\
\hline $\mathrm{DBP}(\mathrm{mmHg})$ & $80.11 \pm 9.29$ & $80.45 \pm 10.12$ & $80.33 \pm 11.26$ & NS & $79.35 \pm 9.81$ & $81.82 \pm 9.82$ & $86 \pm 12.37$ & NS \\
\hline TC (mM) & $5.08 \pm 1.14$ & $4.8 \pm 1.23$ & $4.42 \pm 1.15$ & NS & $4.90 \pm 1.19$ & $4.85 \pm 1.23$ & $4.90 \pm 1.80$ & NS \\
\hline LDL-C (mM) & $3.13 \pm 1.02$ & $2.94 \pm 1.02$ & $2.63 \pm 0.63$ & NS & $2.98 \pm 1.01$ & $2.95 \pm 1.02$ & $2.97 \pm 1.06$ & NS \\
\hline HDL-C (mM) & $1.16 \pm 0.34$ & $1.17 \pm 0.44$ & $1.29 \pm 0.39$ & NS & $1.14 \pm$ & $1.23 \pm 0.52$ & 1.46 & NS \\
\hline $\mathrm{TG}(\mathrm{mM})$ & $1.41 \pm 0.60$ & $1.37 \pm 0.72$ & $1.21 \pm 0.43$ & NS & $1.30 \pm 0.60$ & $1.49 \pm 0.79$ & $1.46 \pm 0.38$ & NS \\
\hline
\end{tabular}

Data are reported as means $\pm \mathrm{SD} . * \mathrm{P}<0.05$. NS $=$ not significant.

\section{DISCUSSION}

In this study, we examined the association of five SNPs with hypertension. Three common $\beta 2$ adrenoceptor gene polymorphisms were evaluated: Arg16Gly (rs1042713), Gln27Glu (rs1042714), and Thr164Ile (rs1800888). These three polymorphisms are all located in the coding region and are known to be functionally important (Leineweber and Brodde, 2004; Brodde, 2008). To the best of our knowledge, this is the first study to examine the association of Arg16Gly, Gln27Glu, and Thr164Ile polymorphisms with the $\beta 2$ adrenoceptor gene in Malaysian hypertensive subjects. All subjects were homozygous for the wild-type allele of the Thr164Ile polymorphism (Thr164), which is consistent with results of Ibrahim et al. (2009), who showed that the allele frequency of the Ile164 mutant among Malays was 0\%; therefore, this SNP was not included in further analyses. For the Arg 16Gly polymorphism, the chi-squared test did not show any significant differences in genotype frequencies between case and control subjects $(\mathrm{P}<0.05)$, and the allele frequencies of Arg16 and Gly16 also did not show any significant difference $(\mathrm{P}=0.062)$. On the other hand, genotype and allele frequencies of Gln27Glu were significantly different between cases and controls $(\mathrm{P}<0.001)$. These findings 
appear to be consistent with another case-control study in an Asian population (Japanese), which found no association between Arg16Gly and hypertension, whereas a significant association was found with the Gln27Glu polymorphism (Kato et al., 2001). A study of the Northern Han Chinese population demonstrated that the Gly16 and Glu27 alleles increased the risk of hypertension (Ge et al., 2005). Studies in other populations, such as Swedish and African-Caribbean, have shown significant associations of the Arg16Gly polymorphism among hypertensive subjects compared to controls (Kotanko et al., 1997; Bengtsson et al., 2001). There are several possible explanations to explain these conflicting results. First, specific ethnic differences could contribute to the association of $\beta 2$ adrenoceptor gene polymorphisms (Xie et al., 1999). Second, there could be linkage disequilibrium or genetic interactions between other alleles of blood pressure regulation genes (Lee et al., 2004). Our study showed that the frequency of Gly16 alleles among hypertensive subjects was $42.5 \%$, which is similar to that of hypertensive subjects in the Japanese population (Kato et al., 2001). We found that only three subjects in each of the case and control groups were homozygous for the mutated allele Glu27. A similar finding was observed in the Japanese population, in which only five subjects among approximately 800 were homozygous for the Glu27 allele (Kato et al., 2001). These findings differed from results obtained from other populations, such as the African-Caribbean population (Kotanko et al., 1997). The Gly16 allele was shown to be more common in Caucasian than Chinese subjects, and the Glu27 allele was more frequent in Caucasian than in AfricanAmerican subjects, and even less frequent among Chinese subjects (Xie et al., 1999).

The $\beta 2$ adrenoceptor is a $\mathrm{G}$ protein-coupled receptor that increases intracellular cAMP of the vascular smooth muscle, resulting in vasodilatation, which in turn lowers the peripheral resistance and hence lowers blood pressure (Pereira et al., 2003). The Gln27Glu polymorphism of the $\beta 2$ adrenoceptor has been studied in relation to hypertension-related conditions such as obesity (Large et al., 1997; Park et al., 2008). In the present study, Gln27Glu was significantly associated with SBP. In a previous study on the Malaysian Malays, the Gln27Glu polymorphism was significantly associated with DBP and some obesity-related parameters (Apalasamy et al., 2011). These results suggested that the Gln27Glu polymorphism of the $\beta 2$ adrenoceptor could be a major risk factor for hypertension and related conditions among Malaysians. As mentioned previously, genotypes of the Arg 16Gly polymorphism of the $\beta 2$ adrenoceptor were not significantly different between cases and controls, whereas those of the Gln27Glu polymorphism were significantly different. We investigated the genotype combination of these two polymorphisms and compared their frequencies between cases and controls, which showed a highly significant difference $(\mathrm{P}<0.001)$. Subjects who were homozygous for both wild-type alleles (Arg16Arg + Gln27Gln) were more frequent in controls $(\mathrm{N}=44 ; 27.5 \%)$ than in cases $(\mathrm{N}=23 ; 14.37 \%)$. Moreover, the heterozygote genotypes were also more common in cases $(\mathrm{N}=66 ; 41.25 \%)$ compared to controls $(\mathrm{N}=23 ; 14.37 \%)$. Finally, none of the study subjects were homozygous for both mutated alleles (Gly16Gly + Glu27Glu). This could be due to the low frequency of the homozygote genotypes of both mutated alleles in our study subjects. The results from the genotype combination analysis suggest that the presence of a mutated allele might increase the risk of hypertension among Malaysians. Thus, the SNP could be in linkage disequilibrium or have interactions with other genes involved in blood pressure regulation.

\section{Limitations}

A number of limitations of the present study should be considered. This study evalu- 
ated the association of only $\beta 2$ adrenoceptor gene polymorphisms. Replication studies with a larger number of subjects and more polymorphisms of other genes are recommended. We also recommend that future studies evaluate the association between these SNPs and hypertension within each race separately. Finally, this study did not evaluate any clinical characteristics related to intermediate phenotypes of hypertension, such as assessing kidney and vascular functions. Assessing these phenotypes and relating them to genotypes could reveal more information about the specific pathways involved in hypertension development.

\section{CONCLUSIONS}

This study revealed an association between the Gln27Glu polymorphism of the $\beta 2$ adrenoceptor in Malaysian hypertensive subjects. Therefore, this polymorphism might be a risk factor associated with hypertension among Malaysians.

\section{ACKNOWLEDGMENTS}

The authors would like to thank the Islamic Development Bank and Genetic Research Group, UPM, for financial support. We also appreciate the assistance of Mr. Mohammed Jokha during sample collection at the Seremban hospital.

\section{Conflicts of interest}

The authors declare no conflict of interest.

\section{REFERENCES}

Agarwal A, Williams GH and Fisher ND (2005). Genetics of human hypertension. Trends Endocrinol. Metab. 16: 127133.

Apalasamy YD, Ming MF, Rampal S, Bulgiba A, et al. (2011). Gender-dependent association of a $\beta 2$ - adrenergic gene variant with obesity parameters in Malaysian Malays. Asia Pac. J. Public Health. Doi: 10.1177/1010539511430250.

Bengtsson K, Orho-Melander M, Melander O, Lindblad U, et al. (2001). $\beta 2$-adrenergic receptor gene variation and hypertension in subjects with type 2 diabetes. Hypertension 37: 1303-1308.

Brodde OE (2008). $\beta-1$ and $\beta-2$ adrenoceptor polymorphisms: functional importance, impact on cardiovascular diseases and drug responses. Pharmacol. Ther. 117: 1-29.

Candy G, Samani N, Norton G, Woodiwiss A, et al. (2000). Association analysis of $\beta 2$ adrenoceptor polymorphisms with hypertension in a Black African population. J. Hypertens. 18: 167-172.

Drysdale CM, McGraw DW, Stack CB, Stephens JC, et al. (2000). Complex promoter and coding region $\beta 2$-adrenergic receptor haplotypes alter receptor expression and predict in vivo responsiveness. Proc. Natl. Acad. Sci. U. S. A. 97: 10483-10488.

Friedewald WT, Levy RI and Fredrickson DS (1972). Estimation of the concentration of low-density lipoprotein cholesterol in plasma, without use of the preparative ultracentrifuge. Clin. Chem. 18: 499-502.

Garland EM and Biaggioni I (2001). Genetic polymorphisms of adrenergic receptors. Clin. Auton. Res. 11: 67-78.

Ge D, Huang J, He J, Li B, et al. (2005). $\beta 2$-adrenergic receptor gene variations associated with stage-2 hypertension in northern Han Chinese. Ann. Hum. Genet. 69: 36-44.

Ibrahim NN, Rasool AH, Wong AR and Rahman AR (2009). Prevalence of $\beta$ - 2 adrenergic receptor ( $\beta 2$ AR) polymorphisms and its influence on a model used to assess endothelial function using pulse wave analysis (PWA). Clin. Chim. Acta 409: 62-66.

Kato N, Sugiyama T, Morita H, Kurihara H, et al. (2001). Association analysis of $\beta 2$-adrenergic receptor polymorphisms with hypertension in Japanese. Hypertension 37: 286-292.

Kotanko P, Binder A, Tasker J, DeFreitas P, et al. (1997). Essential hypertension in African Caribbeans associates with a 
variant of the $\beta 2$-adrenoceptor. Hypertension 30: 773-776.

Large V, Hellstrom L, Reynisdottir S, Lonnqvist F, et al. (1997). Human $\beta-2$ adrenoceptor gene polymorphisms are highly frequent in obesity and associate with altered adipocyte $\beta-2$ adrenoceptor function. J. Clin. Invest. 100: 3005-3013.

Lee YW, Oh VM, Garcia E, Taylor EA, et al. (2004). Haplotypes of the $\beta 2$-adrenergic receptor gene are associated with essential hypertension in a Singaporean Chinese population. J. Hypertens. 22: 2111-2116.

Leineweber $\mathrm{K}$ and Brodde OE (2004). $\beta 2$-adrenoceptor polymorphisms: relation between in vitro and in vivo phenotypes. Life Sci. 74: 2803-2814.

Masuo K, Katsuya T, Fu Y, Rakugi H, et al. (2005). $\beta 2$ - and $\beta 3$-adrenergic receptor polymorphisms are related to the onset of weight gain and blood pressure elevation over 5 years. Circulation 111: 3429-3434.

Park HS, Shin ES and Lee JE (2008). Genotypes and haplotypes of $\beta 2$-adrenergic receptor and parameters of the metabolic syndrome in Korean adolescents. Metabolism 57: 1064-1070.

Pereira AC, Floriano MS, Mota GF, Cunha RS, et al. (2003). $\beta 2$ adrenoceptor functional gene variants, obesity, and blood pressure level interactions in the general population. Hypertension 42: 685-692.

Sethi AA, Tybjaerg-Hansen A, Jensen GB and Nordestgaard BG (2005). 164Ile allele in the $\beta 2$-adrenergic receptor gene is associated with risk of elevated blood pressure in women. The Copenhagen City Heart Study. Pharmacogenet. Genomics 15: 633-645.

Xie HG, Stein CM, Kim RB, Xiao ZS, et al. (1999). Frequency of functionally important $\beta$-2 adrenoceptor polymorphisms varies markedly among African-American, Caucasian and Chinese individuals. Pharmacogenetics 9: 511-516. 